\title{
A second-order theory for transverse ion heating and momentum coupling due to electrostatic ion cyclotron waves
}

\author{
Ronald H. Miller \\ Department of Atmospheric, Oceanic and Space Sciences, Space Physics Research Laboratory, \\ The University of Michigan, Ann Arbor, Michigan 48109-2143 \\ Dan Winske \\ Applied Theoretical Physics Division. Los Alamos National Laboratory, Los Alamos, New Mexico 87545 \\ S. Peter Gary \\ Space and Science Technology Division, Los Alamos National Laboratory, Los Alamos, \\ New Mexico 87545
}

(Received 2 December 1991; accepted 15 May 1992)

\begin{abstract}
A second-order theory for electrostatic instabilities driven by counterstreaming ion beams is developed which describes momentum coupling and heating of the plasma via wave-particle interactions. Exchange rates between the waves and particles are derived, which are suitable for the fluid equations simulating microscopic effects on macroscopic scales. Using a fully kinetic simulation, the electrostatic ion cyclotron instability due to counterstreaming $\mathrm{H}^{+}$beams has been simulated. A power spectrum from the kinetic simulation is used to evaluate second-order exchange rates. The calculated heating and momentum loss from second-order theory is compared to the numerical simulation.
\end{abstract}

\section{INTRODUCTION}

Ion beams streaming with supersonic velocity through a plasma are found in many magnetospheric and space physics environments such as the polar wind, ${ }^{1}$ the auroral region, ${ }^{2}$ the solar wind, ${ }^{3}$ upstream of the Earth's bow shock, ${ }^{4}$ within the bow shock, ${ }^{5}$ the boundary layer of the Earth's magnetotail, ${ }^{6}$ plasmasphere during refilling, ${ }^{7}$ the cometary environment, ${ }^{8}$ and the Jovian ${ }^{9}$ and Saturnian ${ }^{10}$ bow shocks. Such situations can give rise to a number of different instabilities, which are driven by the free energy in the beams and in turn can heat the beams and the background plasma. For example, ion beams propagating parallel to the average magnetic field, $\mathbf{B}_{0}$, have been shown to be unstable to electrostatic ${ }^{1-17}$ and to electromagnetic ${ }^{16,18}$ ion cyclotron instabilities as well as ion-acoustic instabilities. ${ }^{6,19-27}$

Electrostatic ion cyclotron waves are destabilized by electrons $^{28}$ or ions ${ }^{11,16,29}$ streaming along a background magnetic field. The electrostatic ion cyclotron instability (EICI) can be grouped into two classifications: (1) current-driven $\mathrm{EICI}^{12,28,30}$ and (2) ion beam-driven EICI. ${ }^{11,16,17,31,32}$ The current-driven EICI is driven by the inverse electron Landau damping of drifting electrons, while the ion beam can destabilize EIC waves by both resonant ${ }^{16,17}$ and nonresonant ${ }^{11}$ interactions. Drummond and Rosenbluth ${ }^{28}$ first looked at the stability properties of the current-driven EICI and a more detailed treatment followed by Kindel and Kennel. ${ }^{12}$ They found that for a wide range of electron-ion temperature ratios, $T_{e} / T_{i}$, the electrostatic ion cyclotron wave is linearly unstable to small currents. Recently, linear stability of the currentdriven EICI in the auroral region ${ }^{33,34}$ found that EICI can provide a possible source of waves and heating observed experimentally. ${ }^{35-38}$ Particle simulations of current-driven EIC waves generated by multiple component beams ${ }^{39-41}$ confirmed the linear theories and showed significant heating of the beam and background plasma.

The ion beam-driven EICI has been studied extensively in the linear regime ${ }^{11,16,17,31}$ and nonlinear regime. ${ }^{32}$ The resonant and nonresonant interaction can destabilize the EIC waves leading to wave growth and plasma heating. The resonant ion beam-driven EICI is driven by inverse Landau damping, which has the lowest threshold; however, the growth rate for this case is smaller than that driven by reactive coupling between beam cyclotron modes and background ion cyclotron modes. ${ }^{16}$ The nonresonant case is characterized by a parallel phase velocity that is roughly half the beam speed, while for the resonant case, the parallel phase velocity is about the beam speed. ${ }^{17} \mathrm{Mi}$ ura et $a .^{32}$ performed a numerical simulation (three species) of the nonresonant ion beam-driven EICI and found that the mean parallel velocity of the beam ions slowed down, while the perpendicular temperature increased. More recently, Chen and Ashour-Abdalla ${ }^{41}$ simulated a multicomponent plasma (five species) and observed the resonant and nonresonant ion beam-driven EICI, which significantly heated the beans and background plasma. The heating, however, was not quantified in terms of a macroscopic coupling coefficient, and the momentum coupling was not addressed.

Because of the small spatial and short temporal scales of particle simulations, macroscopic fluid simulations (global-scale models) are necessary in order to model large-scale regions such as the polar wind, the auroral region, the solar wind, etc. However, the effects of waveparticle interactions are not included in the development of the fluid equations and hence must be included through coupling coefficients. Palmadesso et $a .^{42}$ developed plasma transport models in the auroral region which included wave-particle interactions and used simplified heating co- 
efficients. The fluid simulation showed that wave-particle effects can change the macroscopic temperature, momentum, and density. A further development of coupling coefficients is needed to include more complex microscopic effects on macroscopic scales.

We consider second-order theory for electrostatic microinstabilities ${ }^{43}$ in a collisionless plasma with one source of free energy (counterstreaming ion beams) to evaluate the momentum and temperature exchange rates for the specific case of the ion beam-driven electrostatic ion cyclotron instability. The calculation assumes a stochastic interaction between the field fluctuations and the particles and that the fluctuation energy density is small compared with the associated species thermal energy. The coefficients are explicit functions of the spectral energy density and hence comparison with numerical simulation is easily carried out, given the spectral energy density. A fully kinetic particle code is then used to simulate a plasma environment which consists of three species: an electron background, and two counterstreaming proton beams. The exchange rates are calculated using the wave energy density from the numerical simulation, and the momentum and temperature variation from second-order theory are compared to the simulation results.

Linear theory is discussed in Sec. II. Second-order theory is discussed and the momentum and energy exchange rates are calculated in Sec. III. The simulation model and results are presented in Sec. IV. In Sec. V we summarize our principal findings and discuss the consequences for ion heating in hydrodynamic simulations.

\section{LINEAR THEORY}

The distribution of the $j$ th species evolves according to the Vlasov equation

$$
\frac{\partial f_{j}}{\partial t}+\mathbf{v} \cdot \frac{\partial f_{j}}{\partial \mathbf{x}}+\frac{e_{j}}{m_{j}}\left(\mathbf{E}(\mathbf{x}, t)+\frac{\mathbf{\nabla} \times \mathbf{B}}{c}\right) \cdot \frac{\partial f_{j}}{\partial \mathbf{v}}=0,
$$

where the magnetic field is assumed to be a constant, and the fluctuation electric field is determined by Poisson's equation,

$$
\nabla \cdot \mathbf{E}=4 \pi \rho(\mathbf{x}, t),
$$

i.e., we assume electrostatic perturbations only. Carrying out a perturbative expansion of the distribution function and the electric field using the magnitude of the fluctuating electric field as an expansion parameter and assuming that $\left|g^{(i+1)}\right|<\left|g^{(i)}\right|$, we obtain

$$
\begin{aligned}
& f_{j}(\mathbf{x}, \mathbf{v}, t)=f_{j}^{(0)}(\mathbf{x}, t)+f_{j}^{(1)}(\mathbf{x}, \mathbf{v}, t)+f_{j}^{(2)}(\mathbf{x}, \mathbf{v}, t)+\cdots \\
& \mathbf{E}(\mathbf{x}, t)=E^{(1)}(\mathbf{x}, t)+E^{(2)}(\mathbf{x}, t)+\cdots .
\end{aligned}
$$

To first order in the expansion parameter $\left(\delta E^{2} / 8 \pi n T\right)$, the Vlasov equation becomes

$$
\frac{\partial f_{j}^{(1)}}{\partial t}+\mathbf{v} \cdot \frac{\partial f_{j}^{(1)}}{\partial \mathbf{x}}=-\frac{e_{j}}{m_{j}} \mathbf{E}^{(1)}(\mathbf{x}, t) \cdot \frac{\partial f_{j}^{(0)}}{\partial \mathbf{v}} .
$$

Integrating Eq. (5) along the particle's unperturbed orbit and taking the zeroth-order velocity moment, we obtain for the first-order density

$$
e_{j} n_{j}^{(1)}(\mathbf{k}, \omega)=-\left[k^{2} \phi^{(1)}(\mathbf{k}, \omega) / 4 \pi\right] K_{j}(\mathbf{k}, \omega),
$$

where we have taken the Fourier and Laplace transform of Eqs. (1)-(5) and where $\phi^{(1)}$ is the electric potential ( $E$ $=-\nabla \phi)$. The plasma susceptibility, $K_{j}$, for the $j$ th species is defined as

$$
\begin{aligned}
K_{j}(\mathbf{k}, \omega)= & -\frac{i \omega_{j}^{2}}{k^{2} n_{j}^{(0)}} \int d^{3} v \int_{-\infty}^{t} d t^{\prime} \mathbf{k} \cdot \frac{\partial f_{j}^{(0)}}{\partial \mathbf{v}} \\
& \times e^{i\left[\mathbf{k} \cdot\left(x^{\prime}-\mathbf{x}\right)-\omega\left(t^{\prime}-t\right)\right]},
\end{aligned}
$$

where $x^{\prime}=x\left(t^{\prime}\right)$ and $v^{\prime}=v\left(t^{\prime}\right)$ represent the unperturbed orbits in the presence of the external fields and where $\omega_{j}$ is the plasma frequency of species $j$. From the linearized Poisson's equation, we obtain the linear dispersion relation

$$
1+\sum_{j} K_{j}(\mathbf{k}, \omega)=0 .
$$

By performing the $t^{\prime}$ integration in Eq. (7), assuming that the zeroth-order distribution satisfies

$$
f_{j}^{(0)}=\frac{n_{j}^{(0)}}{(2 \pi)^{3 / 2} v_{j} v_{j \perp}^{2}} \exp \left[-\frac{1}{2 v_{j}^{2}}\left(\left(v_{\|}-U_{j}\right)^{2}+\frac{T_{j \|}}{T_{j \perp}} v_{\perp}^{2}\right)\right],
$$

and considering a uniform magnetic field $B=B_{0} \mathbf{z}$, the susceptibility can be written in a more familiar form:

$$
\begin{aligned}
K_{j}(\mathbf{k}, \omega)= & \frac{k_{j}^{2}}{k^{2}}\left(1+\sum_{m=-\infty}^{\infty} \frac{\left(\omega-m \Omega_{j} \tau_{j}-\mathbf{k} \cdot U_{j}\right)}{\sqrt{2} k_{z} v_{j}}\right. \\
& \times e^{\left.-\lambda_{j} I_{m}\left(\lambda_{j}\right) Z\left(\zeta_{j}^{m}\right)\right)},
\end{aligned}
$$

where

$$
\begin{aligned}
& \zeta_{j}^{m}=\left(\omega-m \Omega_{j}-k \cdot U_{j}\right) / \sqrt{2} k_{z} v_{j}, \quad \tau_{j}=1-T_{j \|} / T_{j \perp}, \\
& \lambda_{j}=k_{1}^{2} v_{j 1}^{2} / \Omega_{j}^{2}=k_{1}^{2} a_{j}^{2} T_{j 1} / T_{j \|},
\end{aligned}
$$

$U_{j}$ is the drift speed, $v_{j}$ is the parallel thermal velocity $\left(T_{j \|}=m_{j} v_{j}^{2}\right), v_{j 1}$ is the perpendicular thermal velocity $\left(v_{j \perp}^{2}=v_{j}^{2} T_{j \perp} / T_{j \|}\right), \Gamma_{m}\left(\lambda_{j}\right)=\exp \left(-\lambda_{j}\right) I_{m}\left(\lambda_{j}\right)$, where $I_{m}$ is a modified Bessel function of order $m, Z(\zeta)$ is the plasma dispersion function, $\Omega_{j}$ is the cyclotron frequency (where $\left.\omega_{c j}=\left|\Omega_{j}\right|\right), n_{j}^{0}$ is the plasma equilibrium density, and $k_{j}$ is the Debye wave vector.

A three-species plasma is considered: [(1) and (2)] two counterstreaming ion beams and (3) charge neutralizing electrons. The calculation is done in the zero momentum frame for arbitrary beam densities and zero current is assumed which implies that $U_{e}=0$ and $U_{1}=n_{2} / n_{1} U_{2}$, where the $U$ 's represent beam velocities parallel $\left(U_{2}\right)$ and antiparallel $\left(U_{1}\right)$ to the ambient magnetic field and where the $n$ 's are the zeroth-order densities of the beam components. The Doppler-shifted frequency of the cyclotron oscillations of beam 1 and beam 2 is given by 


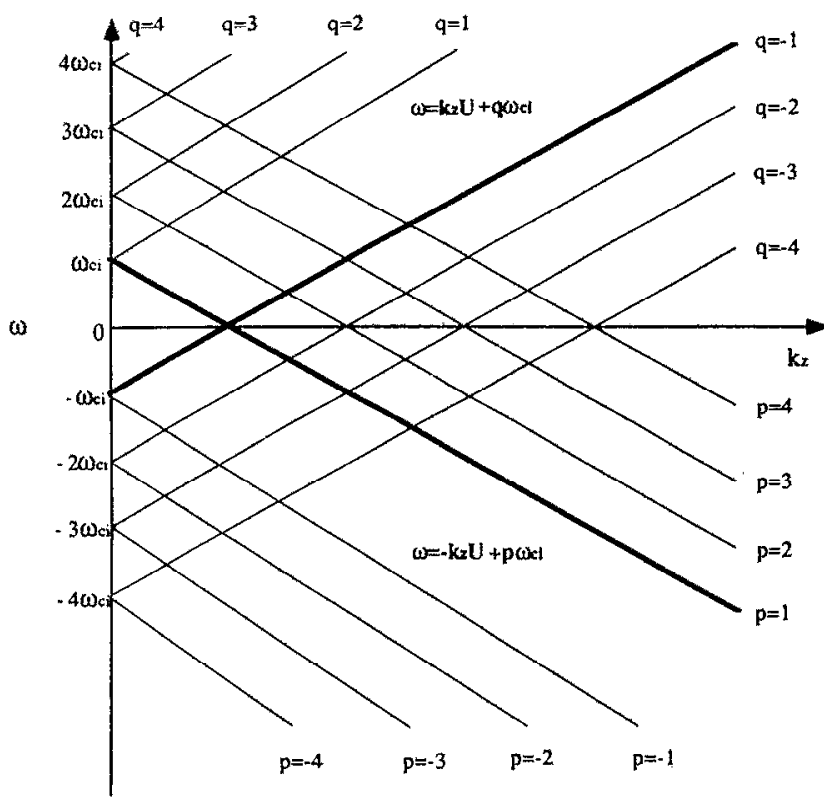

FIG. 1. Counterstreaming ion beam modes as a function of $k_{z}$. The highlighted lines are unstable crossing for $m_{i} / m_{e}=100$ between two of the beam modes, $(p, q)=[1,-(1, \ldots, 5)]$ and $(p, q)=[(1, \ldots, 7),-1]$.

$$
\begin{aligned}
& \omega=-k_{z} U_{1}+p \omega_{c i}, \\
& \omega=k_{z} U_{2}+q \omega_{c i},
\end{aligned}
$$

where $p$ and $q$ are integers and denote gyrofrequency harmonics. When the two frequencies are equal, there is a coupling between the beams that drives the oscillations (normal modes) unstable. Equating Eqs. (11) and (12), the wave vector

$$
k_{z} \approx \omega_{c i}(p-q) /\left(U_{1}+U_{2}\right)
$$

and real frequency

$$
\omega \approx \omega_{c i}\left(q U_{1}+p U_{2}\right) /\left(U_{1}+U_{2}\right)
$$

are obtaincd. Figure 1 shows the intersection between Eqs. (11) and (12) for $q= \pm|n|$ and $p= \pm|m|$, where $n$ and $m$ are integers. The instabilities are generated at these intersections for the wave vector and frequency approximately given by Eqs. (13) and (14), respectively. Transforming into the reference frame of beam 1 , the phase velocity of the instability is $\omega / k_{2}=p\left(U_{1}+U_{2}\right) /(p-q)$ where for $q=0$ the resonant interaction ${ }^{17,32}$ is found for $(p, q)=[(1,2, \ldots, m), 0]$ intersections. For $p=0$, the resonant interaction is found at the $(p, q)=0[(1,2, \ldots, n)]$ crossings (this can be seen by transforming into the beam 2 rest frame): these interactions are sometimes referred to as cyclotron/acoustic $[(1, \ldots, m), 0]$ or acoustic/cyclotron $[0,(1, \ldots, n)]$ modes. The cyclotron/cyclotron modes are when $p$ and $q$ are nonzero. Here, the cyclotron/cyclotron modes include resonant and nonresonant interactions. Perkins ${ }^{16}$ demonstrated that the resonant interaction has the lowest threshold; however, the resonant interaction usually has a smaller growth rate. ${ }^{16,17}$

Numerical solutions of Eq. (8) for the real and imaginary frequency as a function of wave vector are displayed

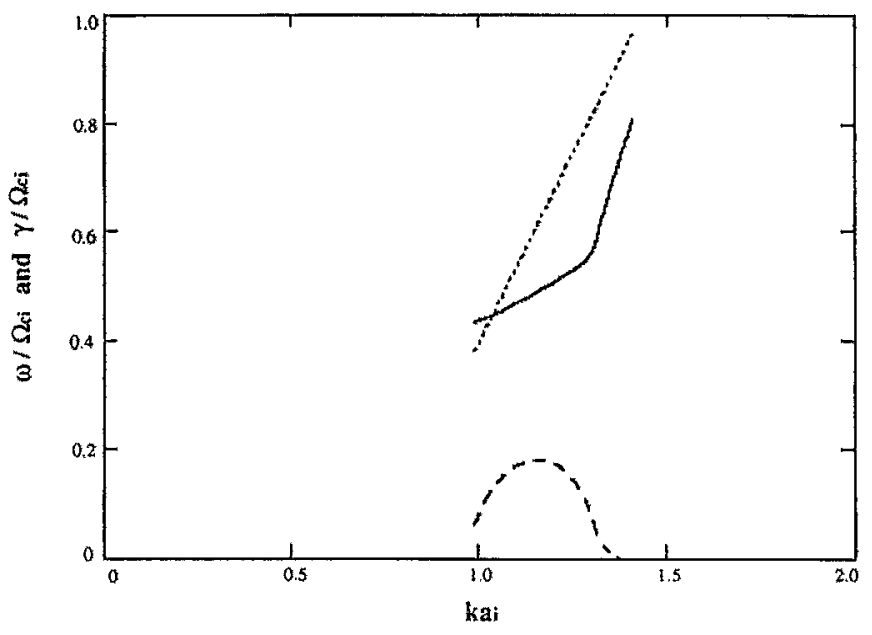

FIG. 2. Linear stability analysis for counterstreaming ion beams for the $(p, q)=(1,-1)$ mode where $m_{i} / m_{e}=1836, \theta=86^{\circ}, \quad n_{1} / n_{e}=0.667$, $n_{2} / n_{e}=0.333, \quad U_{1} / v_{1}=-10.0, \quad U_{2} / v_{2}=20.0, T e / T_{i}=4, \quad \omega_{p e} / \omega_{c e}=0.5, \theta$ $=80^{\circ}$, and $v_{1}=v_{2}$. The real frequency and growth rate, normalized to the ion gyrofrequency, are shown as a function of normalized wave vector $k a_{r}$. The parameters used for the stability analysis remain the same throughout the paper except for the mass ratio, $m_{i} / m_{e}$ and propagation angle.

in Fig. 2, where we have assumed $T_{e} / T_{i}=4, n_{2} / n_{1}=0.5$, $U_{2} / v_{2}=20, U_{1} / v_{1}=-10, \theta=86^{\circ}$, and $m_{i} / m_{e}=1836$. Figure 2 shows the nonresonant ion cyclotron/cyclotron instability $[(p, q)=(1,-1)]$ with a growth rate of $\gamma / \Omega_{p}=0.2$. Two real frequencies are plotted as a function of wave vector in Figure 2: (1) the solid curve which is obtained numerically from Eq. (8) and (2) the dashed curve which is given by Eq. (12). The slopes of the two curves (phase velocity) differ at the peak of the growth rate and become equal as the growth rate decreases. The strong coupling of the two beam modes leads to a variation in the phase velocity of the wave and hence the real frequency. For $80^{\circ}$, the growth rate for the $(p, q)=(1,-1)$ mode $\left(\gamma / \Omega_{p}=0.05\right)$ is reduced by a factor of 4 , while both the real frequency given by Eq. (12) and the numerical solution [Eq. (8)] are approximately equal.

The particle simulation we will discuss later uses a mass ratio of $m_{i} / m_{e}=100$ rather than 1836 , which changes the linear theory result presented in Fig. 2. For a mass ratio of $m_{i} / m_{e}=100$ and $\theta=80^{\circ}$, Figs. $3\left(\omega_{r}>0\right)$ and 4 $\left(\omega_{r}<0\right)$ show the real frequency and growth rates obtained from linear theory (all other parameters remain the same). The reduction in propagation angle, $\theta=80^{\circ}$, produces the same "bell-shaped" growth rate as that obtained for $m_{i} / m_{e}=1836$ at $\theta=86^{\circ}$. The cyclotron/cyclotron modes for positive frequency (Fig. 3) are given by $(p, q)$ $=[(1, \ldots, 7),-1]$, which are nonresonant. The largest growth rate, $\gamma / \Omega_{p}=0.15$, is associated with $(p, q)=(4$, -1 ). The beam mode for $q=-1$, Eq. (12), is plotted in Fig. 3 along with the real frequency computed numerically; both real frequencies have approximately the same linear slope. The bold curve with positive slope in Fig. 1 can be identified as this unstable branch $(p, q)=[(1,2, \ldots, m),-1]$, 


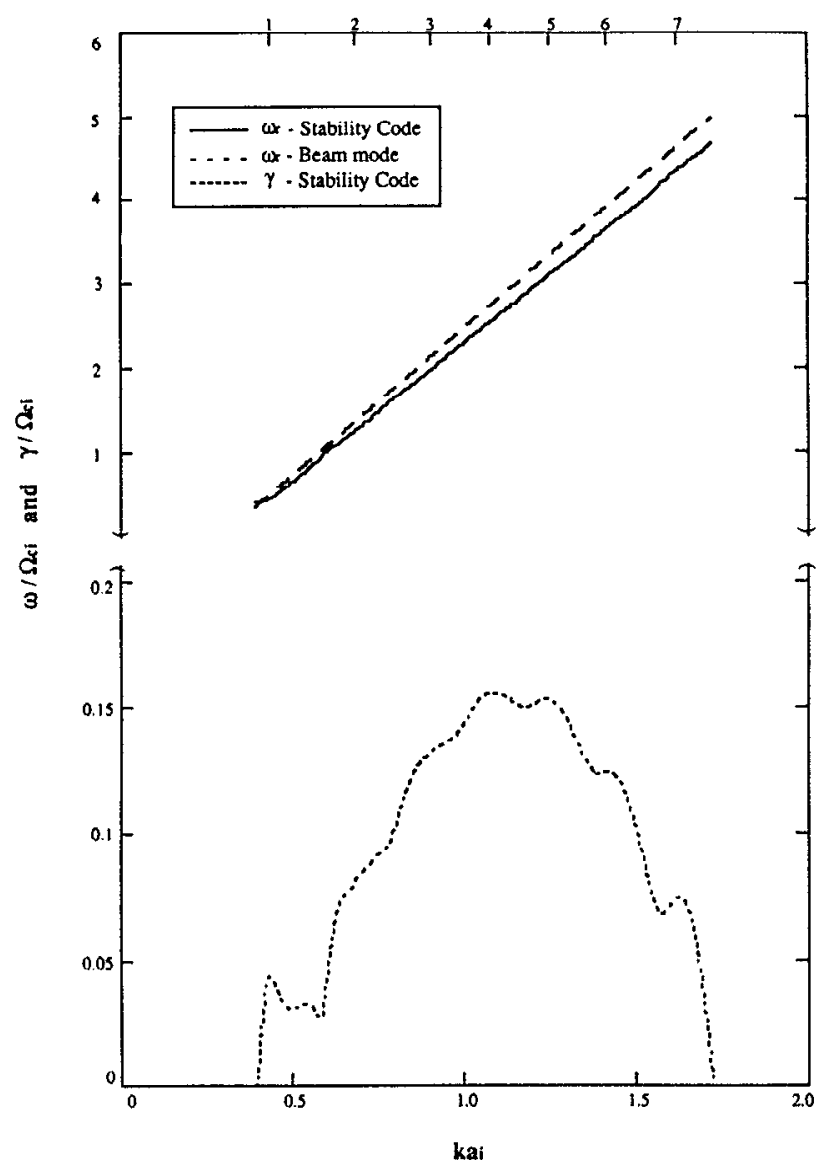

FIG. 3. Linear stability analysis for counterstreaming ion beams for positive real frequency $((p, q)=[(1, \ldots, 7),-1])$ for $m_{i} / m_{e}=100$ and $\theta=80^{\circ}$. The real frequency and growth rate, normalized to the ion gyrofrequency, are shown as a function of normalized wave vector $k a_{i}$

while the other branches with positive slopes $(p, q)$ $=[(1, \ldots, m),-(2, \ldots, n)]$ are stabilized for $m_{i} / m_{e}=100$.

Figure 4 shows the corresponding unstable negative frequency branch $((p, q)=[1,-(3,4,5)])$ for the $m_{i} / m_{e}=100$ case, with the maximum growth rate given by $\gamma / \Omega_{p}=0.03$ associated with $(p, q)=(1,-4)$. The growth rate for $\omega_{r}<0$ is an order of magnitude smaller than that found in Fig. 3. Hence, the waves with positive phase and group velocities (Fig. 3 ) are likely to dominate. The bcam mode with $p=1$, Eq. (11), has approximately the same slope for the real frequency as the numerical results shown in Fig. 3. The bold curve with negative slope in Fig. 1 can be identified as this unstable branch $(p, q)=[1,-(3,4,5)]$, whereas in the case for positive frequency the other branches with negative slopes $(p, q)=[(2, \ldots, m),-(2, \ldots, n)]$ are stabilized for $m_{i} / m_{e}=100$.

The electrostatic ion cyclotron instability saturates for large values of the perpendicular to parallel temperature ratio $T_{1} / T_{\|}$. Using the same parameters as in Figs. 3 and 4, Fig. 5 shows the saturation of the EICI for two wave vectors, $k a_{i}=0.43$ (solid) and 1.12 (dashed). The largest growth rate $\left(\gamma / \Omega_{p} \approx 0.15\right)$ in Fig. 3 corresponds to $k a_{i}=1.12$ which saturates for $T_{\perp} / T_{\|} \approx 3$. For longer wave-

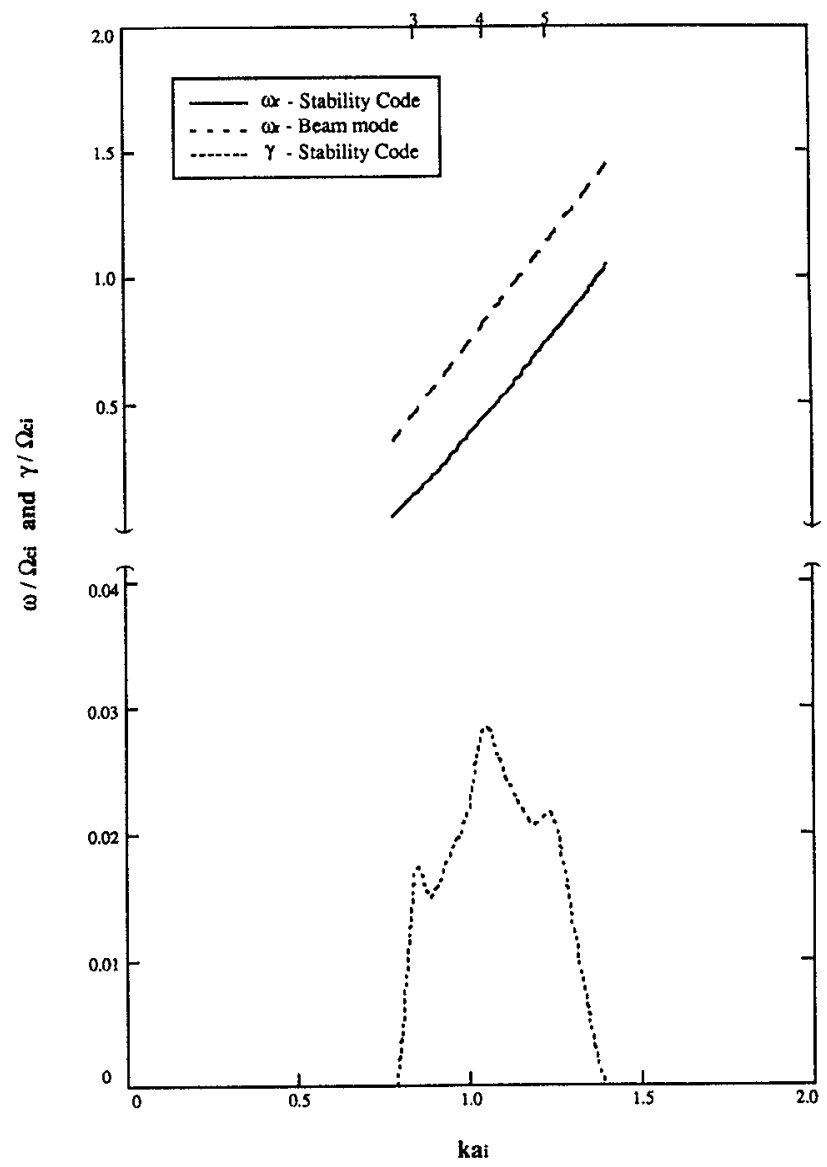

FIG. 4. The normalized real frequency and growth rate for negative real frequency $(p, q)=[1,-(3,4,5)]$ as a function of wave vector for $m_{i} / m_{e}=100$ and $\theta=80^{\circ}$.

lengths, $k a_{i}=0.43$, the growth rate is smaller $\left(\gamma / \Omega_{p} \approx 0.05\right)$, however, the instability saturates at much larger temperature anisotropies, $T_{1} / T_{\|} \approx 10$. This tendency will be seen in the particle simulation as a shift in the dominant wave vector to longer wavelengths.

\section{SECOND-ORDER THEORY}

The second-order exchange rates are easily derived from the moments of the Vlasov equation. Expanding the Vlasov equation to second order and taking an ensemble average over a spectrum of waves, one obtains for a homogeneous plasma ${ }^{43}$

$$
\begin{aligned}
& \frac{\partial\left\langle f_{j}^{(2)}\right\rangle}{\partial t}+\frac{e_{j}}{m_{j}} \frac{\mathbf{v} \times \mathbf{B}_{0}}{c} \cdot \frac{\partial\left\langle f_{j}^{(2)}\right\rangle}{\partial \mathbf{v}} \\
& =-\frac{e_{j}}{m_{j}}\left\langle\mathbf{E}^{(1)}(\mathbf{x}, t) \cdot \frac{\partial f_{j}^{(1)}}{\partial \mathbf{v}}\right\rangle-\frac{e_{j}}{m_{j}}\left\langle\mathbf{E}^{(2)}(\mathbf{x}, t)\right\rangle \cdot \frac{\partial f_{j}^{(0)}}{\partial \mathbf{v}},
\end{aligned}
$$

where $B_{0}$ is the background magnetic field and \langle\rangle represents an ensemble average over the wave field. A necessary criterion for the validity of Eq. (15) is the assumption that the wave-particle interaction is stochastic so one can av- 
Normalized Growth Rates

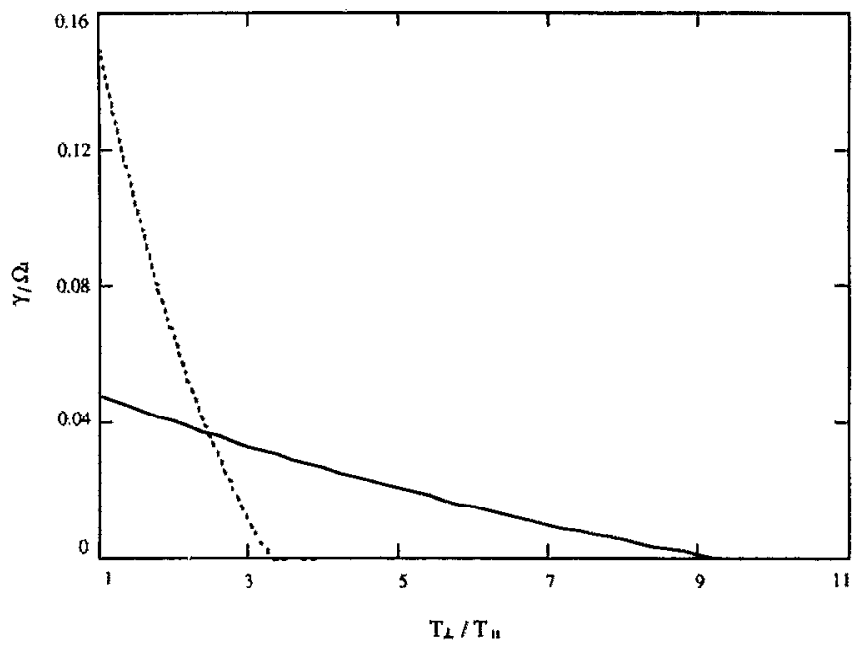

FIG. 5. Growth rate as a function of temperature anisotropy for two wave vectors, $k a_{i}=0.43$ and 1.12 , for $m_{i} / m_{e}=100$ and $\theta=80^{\circ}$.

erage over an ensemble of fluctuations with random phases. Equation (15) describes the slowly varying response to the fluctuating fields during the linear growth phase and is assumed to describe the broadly resonant species response after saturation.

The velocity moments of Eq. (15) yield macroscopic equations that describe the evolution of the second-order density $\left(n_{j}^{(2)}\right)$, momentum density $\left(P_{j}^{(2)}\right)$, and energy density $\left[\left(n_{j} T_{j}\right)^{(2)}\right]$ for the $j$ th species. The first three velocity moments of Eq. (15) can be written as

$\frac{\partial\left\langle n_{j}^{(2)}\right\rangle}{\partial t}=0$,

$\frac{\partial\left\langle\mathbf{P}_{j}^{(2)}\right\rangle}{\partial t}+\mathbf{\Omega}_{j} \times\left\langle\mathbf{P}_{j}^{(2)}\right\rangle-e_{j} n_{j}\left\langle\mathbf{E}^{(2)}\right\rangle=e_{j}\left\langle\mathbf{E}^{(1)} n_{j}^{(1)}\right\rangle$,

$\frac{3}{2} \frac{\partial\left\langle n_{j} T_{j}^{(2)}\right\rangle}{\partial t}=e_{j}\left\langle\mathbf{E}^{(1)} \cdot \Gamma^{(1)}\right\rangle-e_{j} \mathbf{U}_{j}\left\langle\mathbf{E}^{(1)} n_{j}^{(1)}\right\rangle$,

where the right-hand sides of Eqs. (17) and (18) represent wave-particle momentum and temperature exchange rates, respectively (note: the ensemble average of the first-order variations is zero). Using the following definition for the momentum and temperature exchange frequencies,

$$
\begin{aligned}
& v_{P_{j}}=\frac{1}{\mathbf{P}_{j}} e_{j}\left\langle\mathbf{E}^{(1)} n_{j}^{(1)}\right\rangle, \\
& v_{T j}=\frac{2}{3} \frac{1}{n_{j} T}\left(e_{j}\left\langle\mathbf{E}^{(1)} \cdot \Gamma^{(1)}\right\rangle-e_{j} \mathbf{U}_{j}\left\langle\mathbf{E}^{(1)} n_{j}^{(1)}\right\rangle\right),
\end{aligned}
$$

Eqs. (17) and (18) become

$$
\begin{aligned}
& \frac{\partial\left\langle\mathbf{P}_{j}^{(2)}\right\rangle}{\partial t}+\mathbf{\Omega}_{j} \times\left\langle\mathbf{P}_{j}^{(2)}\right\rangle-e_{j} n_{j}\left\langle\mathbf{E}^{(2)}\right\rangle=v_{P j} \mathbf{P}_{j}, \\
& \frac{\partial\left\langle n_{j} T_{j}^{(2)}\right\rangle}{\partial t}=v_{T j} n_{j} T_{j},
\end{aligned}
$$

where the source terms driving the temporal variation of second-order quantities are given in terms of the zerothorder stationary quantities $\left(n_{j} P_{j}, T_{j}\right)$ and their corresponding exchange frequencies $\left(v_{P j}\right.$ and $\left.v_{T j}\right)$. Integrating Eqs. (21) and (22) yields linear temporal growth $\left(v_{P j}>0\right.$, $\left.v_{T j}>0\right)$ or decay $\left(v_{P j}<0, v_{T j}<0\right)$ of the second-order momentum and temperature.

The momentum exchange rate for the $j$ th species is calculated using Eq. (6) and $E^{(1)}=-i k \phi^{(1)}$ for the electric field $^{43}$ yielding

$$
e_{j}\left\langle\mathbf{E}^{(1)} n_{j}^{(1)}\right\rangle=2 \sum_{\mathbf{k}} \mathbf{k} \varepsilon(\mathbf{k}, \omega) \operatorname{Im}\left[K_{j}(\mathbf{k}, \omega)\right],
$$

where $\varepsilon$ is the energy density of the fluctuating fields. The momentum exchange frequency is then given by

$$
v_{P j}=\frac{2}{\mathbf{P}_{j}} \sum_{\mathbf{k}} \mathbf{k} \varepsilon(\mathbf{k}, \omega) \operatorname{Im}\left[K_{j}(\mathbf{k}, \omega)\right],
$$

where we have used Eq. (19). From the zeroth velocity moment of the linearized Vlasov equation it follows directly that

$$
e_{j}\left\langle\mathbf{E}^{(1)} \cdot \Gamma_{j}^{(1)}\right\rangle=2 \sum_{\mathbf{k}} \varepsilon(\mathbf{k}, \omega) \operatorname{Im}\left[\omega K_{j}(\mathbf{k}, \omega)\right]
$$

and therefore the temperature exchange rate ${ }^{43}$ becomes

$$
v_{T j}=\frac{4}{3} \frac{1}{n_{j} T_{j}} \sum_{\mathbf{k}} \varepsilon(\mathbf{k}, \omega) \operatorname{Im}\left[\left(\omega-\mathbf{k} \cdot \mathbf{U}_{j}\right) K_{j}(\mathbf{k}, \omega)\right],
$$

where we have used Eq. (20). The exchange frequencies are given in terms of the plasma dispersion function, which in some regimes can be simplified leading to analytic expressions for the exchange frequencies in terms of macroscopic variables such as the particle density $\left(n_{j}\right)$, momentum $\left(P_{j}\right)$, flow speed $\left(U_{j}\right)$, temperature $\left(T_{j}\right)$, and spectral energy density $(\varepsilon)$. Equation $(22)$ is a scalar equation describing the total temperature increase/decrease due to wave-particle interactions which is easily related to the perpendicular and parallel temperatures, $T=1 / 3\left(T_{\|}\right.$ $+2 T_{1}$ ) (one degree of freedom parallel and two degrees of freedom perpendicular to the magnetic field). For obliquely propagating waves, the formalism does not separate easily into perpendicular and parallel temperatures. However, since the propagation angle is near $90^{\circ}$, most of the heating will be in the perpendicular direction; therefore, Eq. (22) along with Eq. (26) approximately describe the perpendicular heating due to wave-particle interactions. For less oblique waves, one must not assume that the heating is predominantly in the perpendicular direction.

The plasma dispersion function, $Z(\xi)$, in the susceptibility can be expanded in a power series for small argument, $|\xi|<1$, or in an asymptotic series for large argument, $|\zeta|>1$, leading to simplified analytic expressions for the exchange frequencies. The imaginary part of the susceptibility for the two beam species for $|\xi| \ll 1$, using Eqs. (13) and (14), can be written as

$$
\operatorname{Im}\left[K_{t}(k, \omega)\right]=\frac{k_{1}^{2}}{k^{2}} \pi^{1 / 2}\left(\frac{\omega+k_{z} U_{1}}{\sqrt{2} k_{z} v_{1}}\right),
$$




$$
\operatorname{Im}\left[K_{2}(k, \omega)\right]=\frac{k_{2}^{2}}{k^{2}} \pi^{1 / 2}\left(\frac{\omega-k_{z} U_{2}}{\sqrt{2} k_{z} v_{2}}\right),
$$

which can be further simplified using Eqs. (11) and (12) for the Doppler-shifted frequency. The momentum exchange rate in the perpendicular direction, $v_{P y j}$ is zero since there is no zeroth-order momentum $\left(P_{y j}=0\right)$ in that direction. However, the exchange frequencies parallel to the ambient magnetic field are nonzero. Using Eqs. (27) and (28) along with Eqs. (11) and (12), the momentum exchange rates are obtained,

$$
\begin{aligned}
\frac{v_{P z 1}}{\omega_{c i}}= & -2 \pi^{1 / 2} \frac{\omega_{1}^{2}}{\omega_{c i}^{2}}\left(\frac{U_{1}+U_{2}}{\sqrt{2} v_{1}}\right)\left(1+\frac{U_{2}}{U_{1}}\right) \\
& \times \cos ^{2} \theta \sum_{\substack{p, q \\
p>q}} \frac{\varepsilon^{p q}(\omega)}{n_{1} T_{1}} \frac{p}{(p-q)^{2}}, \\
\frac{v_{P z 2}}{\omega_{c i}}= & 2 \pi^{1 / 2} \frac{\omega_{2}^{2}}{\omega_{c i}^{2}}\left(\frac{U_{1}+U_{2}}{\sqrt{2} v_{2}}\right)\left(1+\frac{U_{1}}{U_{2}}\right) \\
& \times \cos ^{2} \theta \sum_{\substack{p, q \\
p>q}} \frac{\varepsilon^{p q}(\omega)}{n_{2} T_{2}} \frac{q}{(p \cdots q)^{2}},
\end{aligned}
$$

where $p$ and $q$ are the mode numbers and $\theta$ is the angle between the magnetic field and the wave vector. The sum is over unstable ion cyclotron wave modes, determined by linear theory, where $p$ and $q$ represent the ion cyclotron harmonics of beams 1 and 2 , respectively. The requirement that $p>q$ yields $k_{z}>0$ from Eq. (13). Similarly, the temperature exchange frequencies are found by computing the imaginary part of the Doppler-shifted frequency times the susceptibility, resulting in

$\frac{v_{T 1}}{\omega_{c i}}=\frac{8}{3} \pi^{1 / 2} \frac{\omega_{1}^{2}}{\omega_{c i}^{2}}\left(\frac{U_{1}+U_{2}}{\sqrt{2} v_{1}}\right)^{3} \cos ^{2} \theta \sum_{\substack{p, q \\ p>q}} \frac{\varepsilon^{p q}(\omega)}{n_{1} T_{1}} \frac{p^{2}}{(p-q)^{3}}$,

$\frac{\nu_{T 2}}{\omega_{c i}}=\frac{8}{3} \pi^{1 / 2} \frac{\omega_{2}^{2}}{\omega_{c i}^{2}}\left(\frac{U_{1}+U_{2}}{\sqrt{2} v_{2}}\right)^{3} \cos ^{2} \theta \sum_{\substack{p, q \\ p>q}} \frac{\varepsilon^{p q}(\omega)}{n_{2} T_{2}} \frac{q^{2}}{(p-q)^{3}}$,

where we have assumed $\gamma^{p q} / \omega_{c i}<1$ and where we have used Eq. (26).

The momentum exchange frequencies in the opposite limit, $|\zeta|>1$, using the following for the imaginary part of the susceptibility:

$$
\begin{aligned}
\operatorname{Im}\left[K_{1}(k, \omega)\right]= & \frac{k_{1}^{2}}{k^{2}}\left(\frac{\gamma}{\omega_{c i}}\right) \frac{T_{1 \|}}{T_{11}} \\
& \times \sum_{m=-\infty}^{\infty} \frac{\Gamma_{m}\left(\lambda_{1}\right) m \omega_{c i}^{2}}{\left(\omega_{r}+k \cos \theta U_{1}-m \omega_{c i}\right)^{2}},
\end{aligned}
$$

$$
\begin{aligned}
\operatorname{Im}\left[K_{2}(k, \omega)\right]= & \frac{k_{2}^{2}}{k^{2}}\left(\frac{\gamma}{\omega_{c i}}\right) \frac{T_{2 \|}}{T_{21}} \\
& \times \sum_{m=-\infty}^{\infty} \frac{\Gamma_{m}\left(\lambda_{2}\right) m \omega_{c i}^{2}}{\left(\omega_{r}-k \cos \theta U_{2}-m \omega_{c i}\right)^{2}},
\end{aligned}
$$

become

$$
\begin{aligned}
\frac{v_{P z 1}}{\omega_{c l}}= & -\sum_{k} 2 \cos \theta \frac{\omega_{1}^{2}}{\omega_{c i}^{2}} \frac{\varepsilon(k, \omega)}{n_{1} T_{1}}\left(\frac{\gamma}{\omega_{c i}}\right)\left(\frac{\omega_{c i}}{k U_{1}}\right) \frac{T_{1 \|}}{T_{11}} \\
& \times \sum_{m=-\infty}^{\infty} \frac{\Gamma_{m}\left(\lambda_{1}\right) m \omega_{c i}^{2}}{\left(\omega_{r}+k \cos \theta U_{1}-m \omega_{c i}\right)^{2}}, \\
\frac{v_{P z 2}}{\omega_{c i}}= & \sum_{k} 2 \cos \theta \frac{\omega_{2}^{2}}{\omega_{c i}^{2}} \frac{\varepsilon(k, \omega)}{n_{2} T_{2}}\left(\frac{\gamma}{\omega_{c i}}\right)\left(\frac{\omega_{c i}}{k U_{2}}\right) \frac{T_{2 \|}}{T_{21}} \\
& \times \sum_{m=-\infty}^{\infty} \frac{\Gamma_{m}\left(\lambda_{2}\right) m \omega_{c i}^{2}}{\left(\omega_{r}-k \cos \theta U_{2}-m \omega_{c i}\right)^{2}},
\end{aligned}
$$

and where the temperature exchange frequencies are given by

$$
\begin{aligned}
\frac{v_{T 1}}{\omega_{c i}}= & \frac{4}{3} \sum_{k} \frac{\omega_{1}^{2}}{\omega_{c i}^{2}} \frac{\varepsilon(k, \omega)}{n_{1} T_{1}}\left(\frac{\gamma}{\omega_{c i}}\right)\left(\frac{\omega_{c i}}{k v_{1}}\right)^{2}\left(1+\frac{T_{1 \|}}{T_{11}}\right. \\
& \left.\times \sum_{m=-\infty}^{\infty} \frac{\Gamma_{m}\left(\lambda_{1}\right) m^{2} \omega_{c i}^{2}}{\left(\omega_{r}+k \cos \theta U_{1}-m \omega_{c i}\right)^{2}}\right), \\
\frac{v_{T 2}}{\omega_{c i}}= & \frac{4}{3} \sum_{k} \frac{\omega_{2}^{2}}{\omega_{c i}^{2}} \frac{\varepsilon(k, \omega)}{n_{2} T_{2}}\left(\frac{\gamma}{\omega_{c i}}\right)\left(\frac{\omega_{c i}}{k v_{2}}\right)^{2}\left(1+\frac{T_{2 \|}}{T_{21}}\right. \\
& \left.\times \sum_{m=-\infty}^{\infty} \frac{\Gamma_{m}\left(\lambda_{2}\right) m^{2} \omega_{c i}^{2}}{\left(\omega_{r}-k \cos \theta U_{2}-m \omega_{c i}\right)^{2}}\right),
\end{aligned}
$$

where knowledge of the relative beam velocity, angle of propagation, temperature, and wave spectral energy density are needed in order to fully evaluate the exchange frequencies.

For the case shown in Fig. $2\left(m_{i} / m_{c}=1836, \theta=86^{\circ}\right)$, the cyclotron/cyclotron instability $[(p, q)=(1,-1)]$ has $|\zeta|=1$ over the range of $k$ space with $\gamma>0$. The dominant contribution to the susceptibility in this case requires a different procedure for the evaluation of the exchange frequencies than those described by Eqs. $(29)-(32)(|\zeta|<1)$ and Eqs. (35)-(37) $(|\zeta|>1)$. Instead, the evaluation of Eqs. (24) and (26) must be done numerically. Figure 6 shows the normalized momentum exchange frequencies $\left[v_{p} /\left(\varepsilon \omega_{c i} / n_{1} T_{1}\right)\right]$ for beam 1 (dashed) and beam 2 (solid) as a function of wave vector. Both momentum exchange frequencies calculated in this manner are negative, with the absolute value used in the graphical display. The negative value of the exchange frequencies implies from Eq. (21) a decrease in the momentum density of each beam due to the generation of ion cyclotron waves. The momentum exchange frequencies have a peaked value of 2.8 for beam 1 and 3.0 for beam 2 and fall off rapidly with $k$. Note that the exchange frequencies are calculated only over the range of $k$ for which $\gamma>0$ (cf. Fig. 2). The temperature exchange frequencies are calculated similarly and are shown in Fig. 7. The temperature exchange frequencies $\left[v_{T} /\left(\varepsilon \omega_{c i} / n_{1} T_{1}\right)\right]$ 
Momentum Exchange Frequencies

$(\omega>0, \mathrm{ml} / \mathrm{me}=1836)$

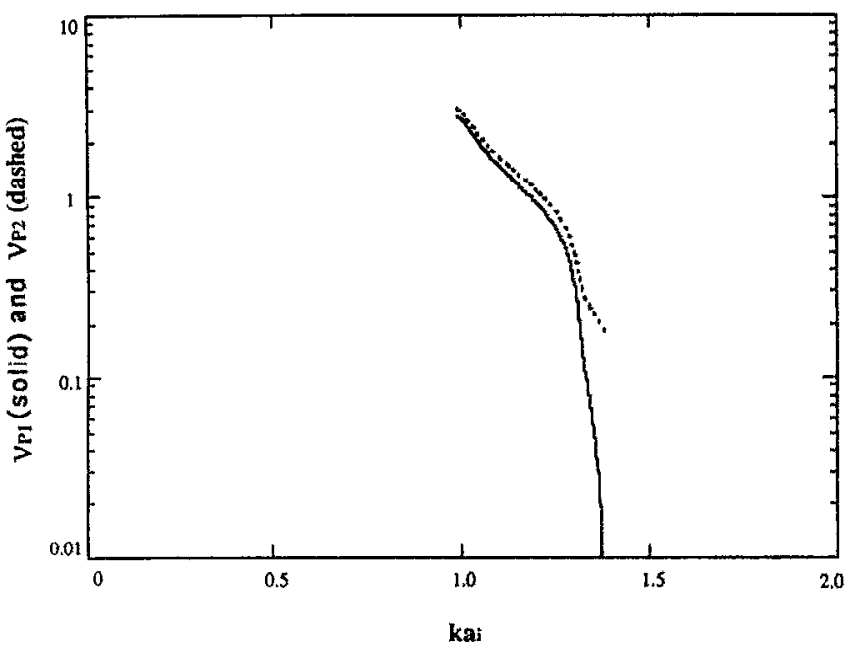

FIG. 6. The second-order momentum exchange frequencies for beam 1 (solid) and beam 2 (dashed) normalized to the gyrofrequency and spectral energy density for $(p, q)=(1,-1)$ as a function of wave vector for $m_{i} / m_{e}=1836$ and $\theta-86^{\circ}$.

are two to three orders of magnitude larger and have the same functional form on $k$ as the momentum exchange frequencies. Beam 2 has a peaked value of $1.15 \times 10^{3}$, which is approximately two times larger than that of beam $1,5.61 \times 10^{2}$.

For the case shown in Figs. 3 and $4\left(m_{i} / m_{e}=100\right.$, $\left.\theta=80^{\circ}\right)$, the exchange rates must be calculated numerically because $|\zeta|=1$ over the entire range of $k$ space with $\gamma>0$. Figure 8 shows the momentum exchange frequencies for

\section{Temperature Exchange Frequencies}

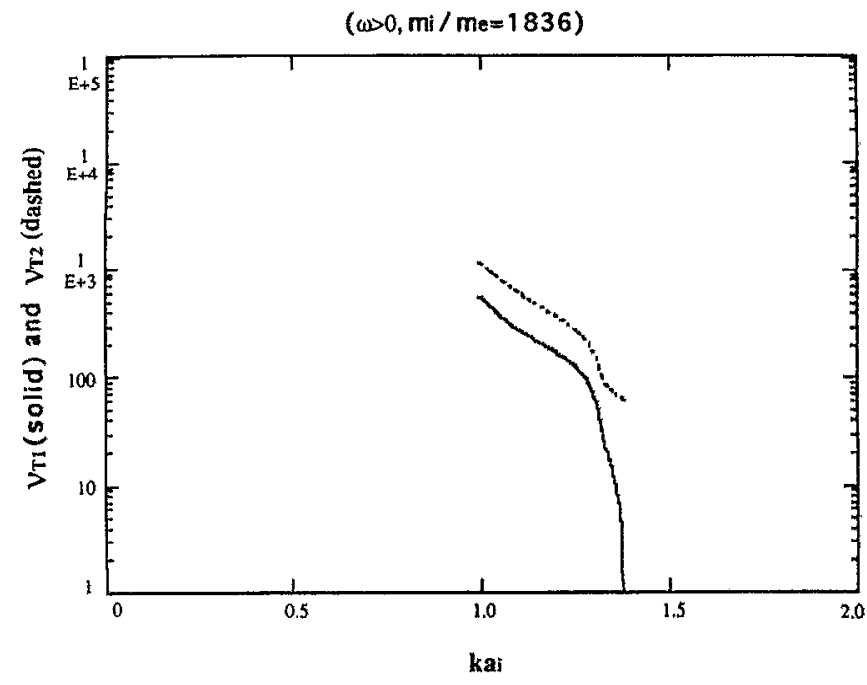

FIG. 7. The second-order temperature exchange frequencies for beam 1 (solid) and beam 2 (dashed) normalized to the gyrofrequency and spectral energy density for $(p, q)=(1,-1)$ as a function of wave vector for $m_{t} / m_{e}=1836$ and $\theta=86^{\circ}$. $(\omega>0, m i / m e=100)$

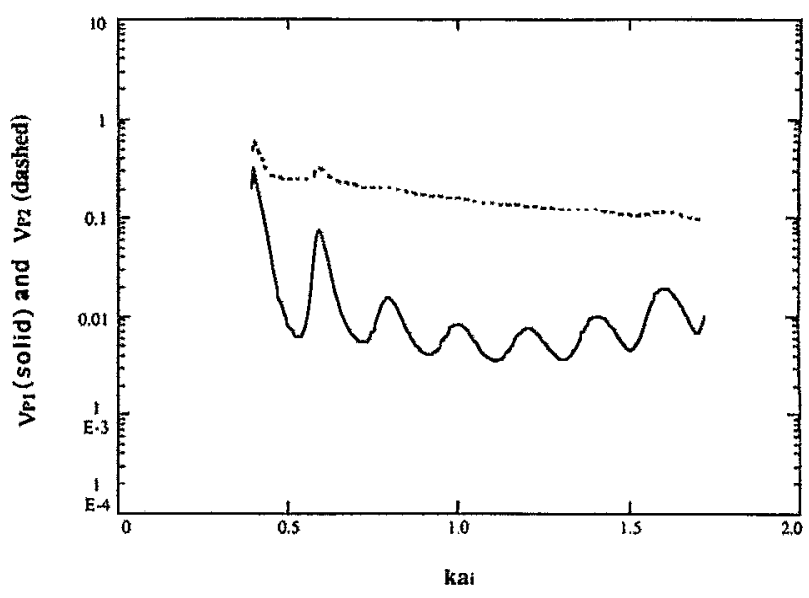

FIG. 8. The second-order momentum exchange frequencies for bean 1 (solid) and beam 2 (dashed) normalized to the gyrofrequency and spectral energy density for positive real frequencies as a function of wave vector for $m_{i} / m_{e}=100$ and $\theta=80^{\circ}$.

the case shown in Fig. 3. $\left(\omega_{r}>0\right)$. Here the range in $k$ over which such frequencies are valid is much broader. The contribution to the momentum exchange frequency for beam 2 [Eq. (12)] is peaked at two cyclotron resonances which correspond to $(p, q)=[(1,2),-1)]$, while beam 1 [Eq. (11)] has several maxima at the crossings of the unstable branch associated with $q=-1$ and higher harmonics, $p=(1, \ldots, 7)$. The momentum exchange frequencies are negative, have a maximum at $(p, q)=(1,-1)$ of 0.27 and 0.53 for beam 1 and beam 2, respectively, and decrease in magnitude at higher $k$.

The contribution of the cyclotron/cyclotron instability for $\omega_{r}<0$ to the momentum exchange frequencies involving $(p, q)=[1,-(3,4,5)]$ is shown in Fig. 9. Beam 2 (solid) asymptotes to a constant value of 0.06 whereas beam 1 (dashed) has peaks corresponding to $(p, q)=(1,-(3,4,5))$ and has a maximum value of 0.013 . These contributions to the momentum exchange frequencies are an order of magnitude smaller than that for the cyclotron/cyclotron instability for $\omega_{r}>0$. Thus, the ion cyclotron waves $\left(\omega_{r}<0\right)$ propagating antiparallel to the magnetic field $\left(v_{g} \| U_{1}\right.$, where $v_{g}$ is the group velocity) do not scatter the beams as effectively as the ion cyclotron waves propagating parallel to the magnetic field $\left(\omega_{r}>0 ; v_{g} \| U_{2}\right)$. The normalized temperature exchange frequencies $\left[v_{T} /\left(\varepsilon \omega_{c i} / n_{1} T_{1}\right)\right]$ for the same case are shown in Fig. 10. Results for positive frequencies are shown in the figure and have approximately the same functional dependence on $k$ as the momentum frequencies (Fig. 8); however, the temperature frequencies are larger in magnitude by two to three orders of magnitude. For beam 1, the normalized temperature exchange frequency have a maximum at $(p, q)=(1,-1)$ of $5.6 \times 10^{1}$ and $2.0 \times 10^{2}$ for beam 1 and beam 2 , respectively. The momentum and temperature exchange frequencies for $m_{i} / m_{e}=1836$ and $\theta=86^{\circ}$ (Figs. 6 and 7) are approximately six to ten times larger than that found for 
Momentum Exchange Frequencies

FIG. 9. The second-order momentum exchange frequencies for beam 1 (solid) and beam 2 (dashed) normalized to the gyrofrequency and spectral energy density for negative real frequencies as a function of wave vector for $m_{f} / m_{e}=100$ and $\theta=80^{\circ}$.

$m_{i} / m_{e}=100$ and $\theta=80^{\circ}$ (Figs. 8 and 10). As with the momentum exchange rates, the negative frequency contributions to the temperature exchange rates are much smaller (factor of 10) and are not explicitly shown here.

To evaluate the exchange rates for the simulation parameters $\left(m_{i} / m_{e}=100\right)$, we keep only the one term in Eqs. (24) and (26), the largest contributors to the exchange frequencies $(p, q)=(1,-1)$. We obtain the following, where $\varepsilon$ is the wave energy in the dominant mode:

$$
\frac{v_{P_{z 1}}}{\omega_{c i}}=-2.7 \times 10^{-1} \frac{\varepsilon}{n_{1} T_{1}},
$$

Temperature Exchange Frequencies

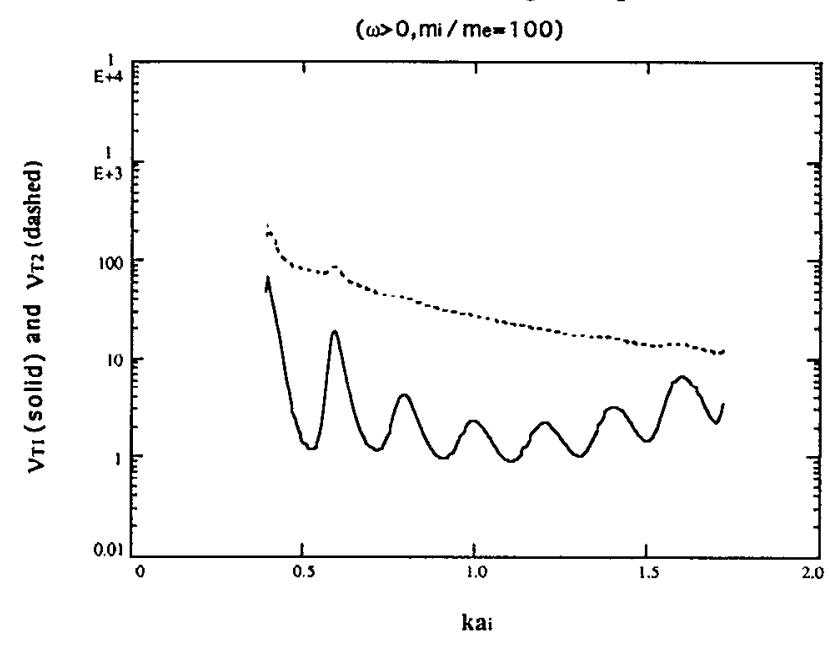

FIG. 10. The second-order temperature exchange frequencies for beam 1 (solid) and beam 2 (dashed) normalized to the gyrofrequency and spectral energy density for positive real frequencies as a function of wave vector for $m_{i} / m_{e}=100$ and $\theta=80^{\circ}$.

$$
\begin{aligned}
& \frac{v_{P z 2}}{\omega_{c i}}=-5.3 \times 10^{-1} \frac{\varepsilon}{n_{1} T_{1}}, \\
& \frac{v_{T 1}}{\omega_{c i}}=5.6 \times 10^{1} \frac{\varepsilon}{n_{1} T_{1}}, \\
& \frac{v_{T 2}}{\omega_{c i}}=2.0 \times 10^{2} \frac{\varepsilon}{n_{1} T_{1}},
\end{aligned}
$$

where knowledge of the energy density is necessary in order to fully evaluate the exchange frequencies. As we have used the peak exchange rates (in $k$ ), we expect these formulas may overestimate the actual exchange frequencies. From Eqs. (39)-(42), one can conclude that beam 2 (fast/tenuous) loses momentum and increases in temperature faster than beam 1 (slow/dense) for a given spectral energy density. To proceed further, the energy density must be calculated, either using experimental data, numerically, or analytically using various approximations. In the following section, the energy density is calculated numerically, and Eqs. (39)-(42) are compared to the numerical simulation results.

\section{SIMULATION}

In this section results of a particle-in-cell simulation of the ion beam-driven electrostatic ion cyclotron instability are presented. The cyclotron/cyclotron instability has been simulated previously by Miura et $a l^{32}$ and more recently by Chen and Ashour-Abdalla ${ }^{41}$ with two ion species $\left(\mathrm{H}^{+}\right.$and $\left.\mathrm{O}^{+}\right)$, each species involving both beam and background components. Unlike the previous calculations that used guiding-center electrons and particle ions, here we carry out fully kinetic simulations using WAVE. ${ }^{44}$ The simulations are done in the electron rest frame which corresponds to the zero momentum frame. The plasma is initially spatially homogeneous in space; the systcm is quasineutral with zero net current. Periodic boundary conditions for the fields and the plasma are assumed, and only electrostatic perturbations are allowed. The simulations has one spatial coordinate, while the fields and particle velocities are calculated in three dimensions.

The calculation uses 10000 electrons and ions distributed uniformly over the simulation domain. The simulation axis is directed along the electric field vector at an angle of $80^{\circ}$ from the magnetic field. The system length is taken to be $4.8 \mathrm{c} / \omega_{p e}$ (electron inertial lengths), approximately six times the wavelength of the dominant instability. The calculation uses 64 computational cells with a time step of $\Delta t \omega_{p e}=0.05$ with $\omega_{p e} / \omega_{c e}=0.5$ and $N_{t}=30000$, where $N_{t}$ is the number of time steps. The drift speeds for the two ion beams are $U_{1} / v_{1}=-10.0$ and $U_{2} / v_{2}=20$ with a normalized beam density of $n_{1} / n_{e}=0.667$ and $n_{2} / n_{e}=0.333$, respectively. The thermal velocity of the electrons is taken as $v_{e} / c=0.05$ and for $m_{i} / m_{e}=100$ and $T_{e} / T_{i}=4$, the ion thermal velocities become $v_{1}=v_{2}$ $=0.0025$. These parameters correspond to those shown in Figs. 3, 4, and 8-10.

Figure 11 shows the time history of the normalized fluctuating electrostatic field energy density averaged over 


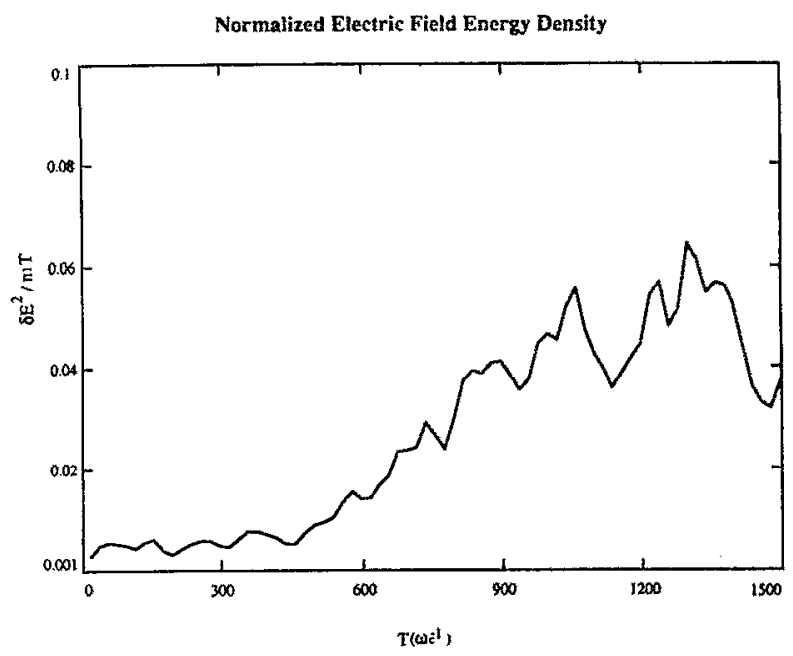

FIG. 11. The fluctuating electric field energy density as a function of time. The numerical simulation uses the same parameters as the linear stability analysis and $m_{i} / m_{e}=100, \theta=80^{\circ}, N_{e}=N_{i}=10000, v_{e} / c=0.05$, $L_{x}=4.8 c / \omega_{p e} N_{x}=64, \Delta t \omega_{p e}=0.05$, and $N_{t}=30000$.

the entire simulation domain, $\delta^{2} E /\left(8 \pi n_{1} T_{1}\right)$. The fluctuation level grows by an order of magnitude from $\delta^{2} E /\left(8 \pi n_{1} T_{1}\right) \approx 0.006$ to 0.06 between approximately 600 to $1200 \omega_{p e}^{-1}$. The initial field growth, $t=600 \omega_{p e}^{-1}$, is primarily in the $N=6$ Fourier mode which corresponds to $(p, q)$ $=(4,-1)$ and has the largest growth rate, consistent with Fig. 3. From 900 to $1200 \omega_{p e}^{-1}$, the dominant mode shifts to longer wavelengths (to $N=3$ ), which corresponds to $(p, q)$ $=(1,-1)$. The fluctuating electric field energy density saturates at $t \approx 1200 \omega_{p e}^{-1}$. During the interval $900-1200 \omega_{p e}^{-1}$ the average normalized spectral energy density for $N=3$ is found to be $\delta^{2} E_{k} /\left(8 \pi n_{1} T_{1}\right) \approx 0.01$. Given this fluctuation level, the second-order theory exchange frequencies [Eqs. (39)-(42)] can be evaluated. During this interval using the corresponding spectral energy density for $N=3$, one finds the momentum exchange rates for the two beams to be $v_{P 1} / \omega_{c i} \approx-2.7 \times 10^{-3}$ and $v_{P 2} / \omega_{c i} \approx-5.3 \times 10^{-3}$. In similar fashion one computes the theoretical temperature exchange rates: $v_{T 1} / \omega_{c i} \approx 0.56$ and $v_{T 2} / \omega_{c i} \approx 2.00$.

Figure 12 shows the average velocity parallel to the magnetic field as a function of time in the simulation where the velocities are normalized to their initial values. The solid and dashed lines correspond to beam 1 (slow/dense) and beam 2 (fast/tenuous), respectively. The background noise in the simulation slightly scatters the beams initially; however, scattering does not become significant until the instability grows out of the noise, which, occurs approximately by $600 \omega_{p e}^{-1}$. From Fig. 12, we see that beam 2 losses momentum at a faster rate than beam 1 , which is consistent with the analytic results from second-order theory [Eqs. (39)-(42)]. Between 900 and $1200 \omega_{p e}^{-1}$ the slopes of the normalized momentum for the ion beams are approximately linear, which corresponds to the regime of applicability of the second-order theory [Eqs. (21) and (22)]. Performing a linear fit for the momentum of the two ion
Parallel Velocity vs Time

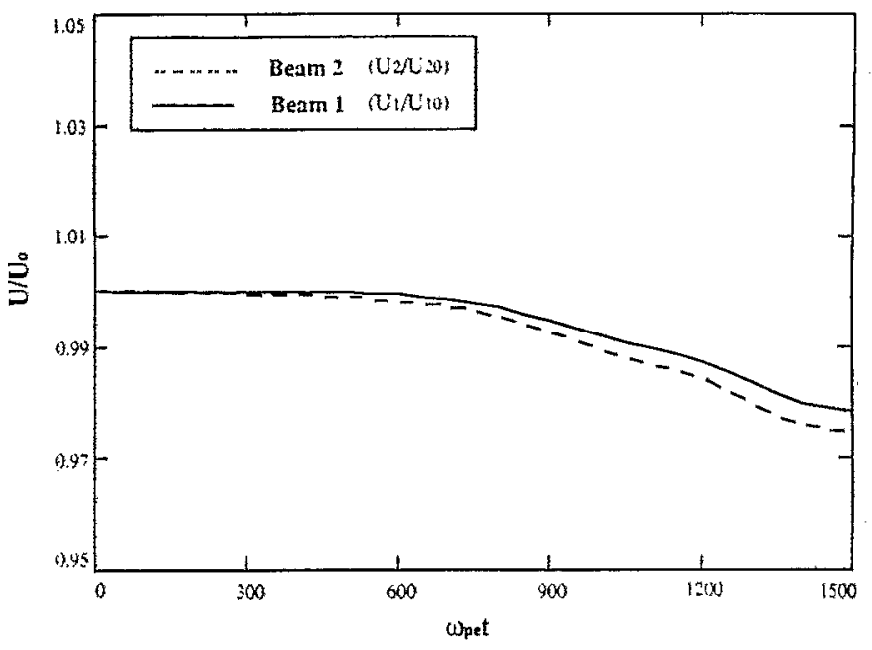

FIG. 12. The normalized momentum for beam 1 (solid) and beam 2 (dashed) as a function of time from the particle simulation.

beams, one obtains the following for the momentum exchange frequencies $v_{P_{1}} / \omega_{c i} \approx-1.22 \times 10^{-3}$ and $v_{P 2} / \omega_{c i}$ $\approx-1.41 \times 10^{-3}$.

The simulation results for perpendicular heating of the counterstreaming ion beams are shown in Fig. 13. As with the parallel velocity, the perpendicular temperatures for the two beams change only by a small amount prior to $600 \omega_{p e}^{-1}$, followed by large changes as the waves grow. The change in the temperature (as with the velocity) is largest for the faster, more tenuous beam. By $1200 \omega_{p e}^{-1}$, the field energy saturates $\left(T_{12} / T_{\| 2} \approx 10\right)$. Linear theory for this case (Fig. 5 ) indicates that the fastest growing mode ( $N$ $=6)$ saturates at a small value of $T_{1} / T_{\|}(=3)$, but that the longer wavelength $(N=3)$ mode continues to grow until $T_{\perp} / T_{\|} \approx 10$. Both the shift in the wavelength and the

Perpendicular Ion Beam Temperatures vs Time

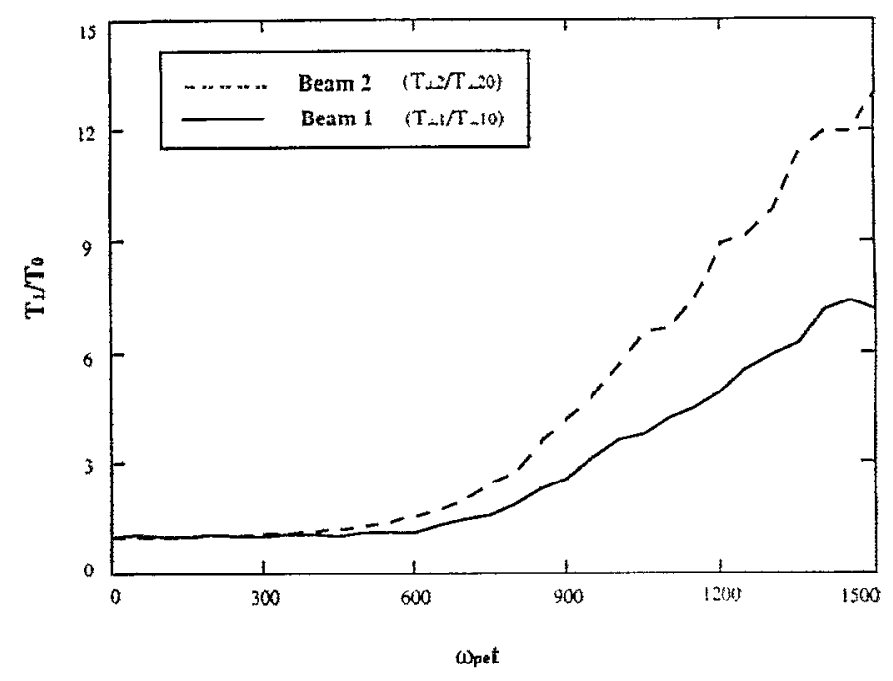

FIG. 13. The normalized temperature for beam 1 (solid) and beam 2 (dashed) as a function of time from the particle simulation. 

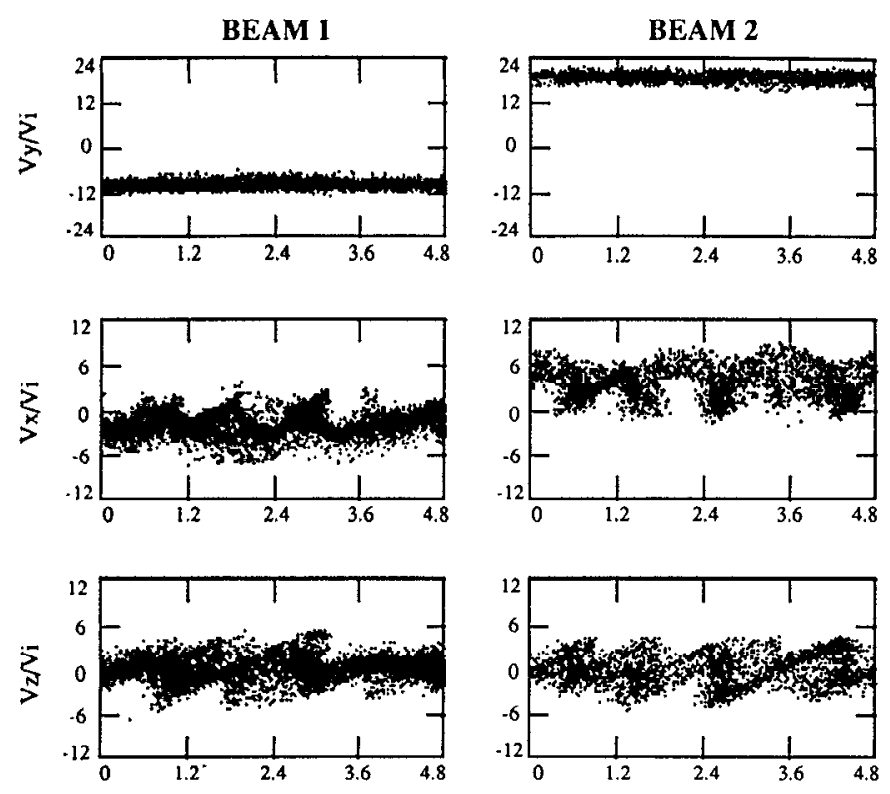

$X\left(c / \omega_{p c}\right)$

$\mathrm{X}\left(\mathrm{c} / \omega_{\mathrm{pc}}\right)$

$\mathrm{t} \omega \mathrm{pe}=1200$

FIG. 14. Ion phase space plots at $\omega_{p e} t=1200$ from the particle simulation.

large value of $T_{1} / T_{\|}$when the fluctuations saturate are consistent with a quasilinear evolution of the system. At later time, heating of both beams continues because of the continued high fluctuation levels after saturation (Fig. 11). This effect is accentuated due to the one-dimensional (1D) nature of the calculation that constrains the decay of the fluctuations. The temperature exchange frequencies are calculated by using a linear fit to the perpendicular temperature of the ion beams. These rates are found to be $v_{T 1} / \omega_{c i} \approx 0.38$ and $v_{T 2} / \omega_{c i} \approx 0.71$.

Finally, to show details of the heating process, in Fig. 14 we plot $v_{y}-x, v_{x}-x$, and $v_{z}-x$ phase space for the two ion beams (beam 1, left panels; beam 2, right panels) at $\omega_{p e c} t=1200$, when the waves saturate. Here $x$ corresponds to the direction of $k$ so that $y$ is nearly along B. In the $v_{y}-x$ plot we see only weak spatial bunching. In the two velocity directions perpendicular to $\mathbf{B}$, however, we see strong ion heating. As in Chen and Ashour-Abdalla ${ }^{41}$ the heating results from coherent trapping motion, as seen by the vortices. The fact that the center of the vortices occurs at $v_{x} \approx U_{1} / 3$ indicates that the instability is "nonresonant" $\left(\omega / k_{\|} \approx V_{b} / 2\right.$ in the background ion frame $\left.{ }^{32}\right)$. From the phase space plots it is also evident that the heating of beam 2 is somewhat larger than that of beam 1 . Some of the coherent features are, of course, an artifact of the 1-D nature of the simulation.

\section{SUMMARY}

In this paper, we have considered second-order theory for electrostatic microinstabilities in a collisionless plasma with one source of free energy (counterstreaming ion beams) and have analytically derived momentum and energy exchange frequencies for the ion beams in two limits:
(1) For $|\zeta|<1$, Eqs. (29) $-(32)$, and (2) for $|\zeta|>1$, Eqs. (35) $-(38)$. Both limits of the coefficients depend on macroscopic parameters such as density, temperature, angle of propagation, magnetic field strength, as well as the fluctuation spectrum. The coefficients can be evaluated at $(p, q)$ $=(1,-1)$ for most applications, which simplifies the expressions by removing the summation over unstable modes.

However, the cyclotron/cyclotron instability simulated in this paper is nonresonant with $|\zeta| \approx 1$, which requires the exact evaluation of the exchange frequencies, Eqs. (24) and (26). In this case, the coefficients, normalized to the gyrofrequency and to the spectral energy density, are thus evaluated numerically. The largest contribution to the second-order theory exchange frequencies, given by $(p, q)=(1,-1)$, yields $v_{P 1} / \omega_{c i} \approx-2.7 \times 10^{-3}$, $v_{P 2} / \omega_{c i} \approx-5.3 \times 10^{-3}, v_{T 1} / \omega_{c i} \approx 0.56$, and $v_{T 2} / \omega_{c i} \approx 2.00$ for the momentum and temperature exchange frequencies for each beam, respectively.

The simulation of the cyclotron/cyclotron instability in Sec. IV shows significant parallel momentum loss (Fig. 12) and heating (Fig. 13) of the two ion beams. Taking a linear fit of the normalized momentum and temperature, the numerically simulated exchange frequencies are found to be $v_{P 1} / \omega_{c i} \approx-1.22 \times 10^{-3}, \quad v_{P 2} / \omega_{c i} \approx-1.41 \times 10^{-3}$, $v_{T 1} / \omega_{c i} \approx 0.38$, and $v_{T 2} / \omega_{c i} \approx 0.71$. The ratio of secondorder theory $(S)$ results to numerical simulation $(N)$ results thus yields $\left(v_{P_{1 S}} / v_{P 1 N}\right)=2.2, \quad\left(v_{P 2 S} / v_{P 2 N}\right)=3.7$, $\left(v_{T 1 S} / v_{T 1 N}\right)=1.5$, and $\left(v_{T 2 S} / v_{T 2 N}\right)=2.8$. Therefore, exchange rate coefficients determined from second-order theory give values that agree to within a factor of approximately 4 to those obtained from the numerical simulation. This range of agreement is acceptable because, as discussed earlier, our theoretical estimates tend to be too large. In addition, in the simulation the spectral energy density is relatively low (i.e., the noise level is relatively high). In this case we expect the noise to act as an effective collision frequency, reducing the growth rates, and corresponding exchange rates, of the instability.

While the phase space dynamics of the simulation presented in Sec. IV suggest that some coherent phenomena (i.e., trapping) occur, the evolution of the fluctuation spectrum to longer wavelengths and the saturation of the instability at $T_{\perp} / T_{\|} \approx 10$ are consistent with quasilinear stabilization. As noted by Miura et al. ${ }^{32}$ two-dimensional simulations of the cyclotron/cyclotron EIC instability yield a broad wave spectrum (again suggesting a more quasilinear evolution), but overall similar final values of macroscopic quantities to those found in 1-D calculations. It is evident that the second-order exchange rates, along with an equation for the time evolution of the fluctuation energy density can be solved together to obtain a selfconsistent quasilinear evolution of the system. Particle simulations, such as the calculations done in this paper, which are much more costly than solving the evolutionary equations, can occasionally be used to check the solutions. Carrying out such quasilinear calculations over a wide range of parameters will then allow the development of transport coefficients that are only a function of macroscopic param- 
eters. These transport coefficients can in turn be used in fluid simulations of polar wind phenomena on long time and large distance scales. The development of such a timedependent quasilinear model to allow the parametrization of these coefficients is our next goal.

\section{ACKNOWLEDGMENTS}

This work was supported at the University of Michigan by National Aeronautics and Space Administration (NASA) Grants No. NAGW-2162 and No. NAGW-1619 and by the National Science Foundation under Contract No. ATM-9114409. Research at Los Alamos was done under the auspices of the U.S. Department of Energy and was supported in part by the National Aeronautics and Space Administration Research and Analysis Program at the Laboratory.

'P. M. Banks and T. E. Holzer, J. Geophys. Res. 74, 6317 (1969).

${ }^{2}$ E. G. Shelley, R. D. Sharp, and R. G. Johnson, Geophys. Res. Lett. 3, 654 (1976).

${ }^{3}$ W. C. Feldman, J. R. Asbridge, S. J. Bame, and M. D. Montgomery, J. Geophys. Res. 78, 2017 (1973).

${ }^{4}$ M. F. Thomsen, "Collisionless Shocks in the Heliosphere: Reviews of Current Research," edited by B. T. Tsurutani and R. G. Stone, Geophysical Monograph 35, 253 (1988).

${ }^{5}$ G. Paschmann, N. Sckopke, S. J. Bame, and J. T. Gosling, Geophys. Res. Lett. 9, 881 (1982).

${ }^{6}$ C. L. Grabbe and T. E. Eastman, J. Geophys. Res. 89, 3865 (1984).

${ }^{7}$ J. J. Sojka and G. L. Wrenn, J. Geophys. Res. 90, 6379 (1985).

${ }^{8}$ G. Gloeckler, D. Hoverstadt, F. M. Ipavich, M. Scholer, B. Klecker, and A. B. Galvin, Geophys. Res. Lett. 13, 251 (1986).

${ }^{9}$ S. L. Moses, F. V. Coroniti, C. F. Kennel, F. L. Scarf, E. W. Greenstadt, W. S. Kurth, and R. P. Lepping, Geophys. Res. Lett. 12, 183 (1985).

${ }^{10}$ D. A. Gurnett, W. S. Kurth, and F. L. Scarf, Science 212, 235 (1981).

${ }^{11}$ E. S. Weibel, Phys. Fluids 13, 3003 (1970).

${ }^{12}$ J. M. Kindel and C. F. Kennel, J. Geophys. Res. 76, 3055 (1971).

${ }^{13}$ T. Stix, Phys. Fluids 16, 1922 (1973).
${ }^{14}$ H. L. Berk, W. Horton, Jr., M. N. Rosenbluth, and P. H. Rutherford, Nucl. Fusion 15, 819 (1975).

${ }^{15}$ B. Coppi and K. Bhadra, Phys. Fluids 18, 692 (1975).

${ }^{16} \mathrm{~F}$. W. Perkins, Phys. Fluids 19, 1012 (1976).

${ }^{17}$ M. Yamada, S. Seiler, H. W. Hendel, and H. Ikezi, Phys. Fluids 20, 450 (1977).

${ }^{18}$ J. M. Cornwall and M. Schulz, J. Geophys. Res. 76, 7791 (1971).

${ }^{19}$ T. E. Stringer, Plasma Phys. 6, 267 (1964).

${ }^{20}$ S. P. Gary, J. Geophys. Res. 83, 2504 (1978)

${ }^{21}$ D. S. Lemons, L. R. Asbridge, S. J. Bame, W. C. Feldman, S. P. Gary, and J. T. Gosling, J. Geophys. Res. 84, 2135 (1979).

${ }^{22}$ R. Bergmann, J. Geophys. Res. 89, 953 (1984).

${ }^{23}$ C. L. Grabbe, Geophys. Res. Lett. 12, 483 (1985).

${ }^{24}$ P. B. Dusenbery and L. R. Lyons, J. Geophys. Res. 86, 7627 (1981).

${ }^{25} \mathrm{~K}$. Akimoto and D. Winske, J. Geopliys. Res. 90, 12095 (1985).

${ }^{26}$ N. Omidi, J. Geophys. Res. 90, 12330 (1985).

${ }^{27} \mathrm{~K}$. Akimoto and N. Omidi, Geophys. Res. Lett. 13, 97 (1986).

${ }^{28}$ W. E. Drummond and M. N. Rosenbluth, Phys. Fluids 7, 1507 (1962).

${ }^{29}$ P. Michelsen, Phys. Fluids 19, 337 (1976).

${ }^{30}$ J. J. Rasmussen and R. W. Schrittwieser, IEEE Trans. 19, 457 (1991).

${ }^{31}$ R. L. Kaufmann and P. M. Kintner, J. Geophys. Res. 87, 10487 (1982).

${ }^{32}$ A. Miura, H. Okuda, and M. Ashour-Abdalla, Geophys. Res. Lett. 10, 353 (1983).

${ }^{33}$ R. Bergmann, I. Roth, and M. K. Hudson, J. Geophys. Res. 93, 4005 (1988).

${ }^{34}$ D. Schriver and M. Ashour-Abdalla, J. Geophys. Res. 93, 2633 (1988).

${ }^{35}$ P. M. Kintner, M. C. Kelley, and F. S. Mozer, Geophys. Res. Lett. 5, 139 (1979).

${ }^{36}$ M. Temerin, F. S. Mozer, and M. Woldorff, Phys. Res. Lett. 43, 1941 (1979).

${ }^{37}$ M. Kintner, M. C. Kelley, R. D. Sharp, A. G. Ghielmetti, M. Temerin, C. Cattell, P. F. Mizera, and J. F. Fennel, J. Geophys. Res. 84, 7201 (1979).

${ }^{38}$ C. Cattell, J. Geophys. Res. 86, 3641 (1981).

${ }^{39}$ K. Okuda and M. Ashour-Abdalla, J. Geophys. Res. 88, 899 (1983).

${ }^{40} \mathrm{M}$. Ashour-Abdalta, H. Okuda, and S. Y. Kim, Geophys. Res. Lett. 14, 375 (1987).

${ }^{41}$ M. Chen and M. Ashour-Abdalla, J, Geophys. Res. 93, 18949 (1990).

${ }^{42}$ P. J. Palmadesso, S. B. Ganguli, and H. G. Mitchell, Jr., "Modeling Magnetospheric Plasma," edited by T. E. Moore and J. H. Waite, Jr,, Geophysical Monograph 44, 133 (1988).

${ }^{43}$ S. P. Gary, Phys. Fluids 23, 1193 (1980).

${ }^{44}$ D. W. Forslund, Space Sci. Rev. 42, 3 (1985). 